\title{
A DECENTRALIZED ADAPTIVE MEDIUM ACCESS CONTROL FOR V2I VANET
}

\author{
Neelambike $\mathbf{S}^{\mathbf{1}}$ and Chandrika $\mathbf{J}^{\mathbf{2}}$ \\ ${ }^{1}$ Department of Information Science and Engineering, GM Institute of Technology, India \\ ${ }^{2}$ Department of Computer Science and Engineering, Malnad College of Engineering, India
}

\begin{abstract}
Vehicular Adhoc Networks (VANETs) resemble similar characteristic as Mobile Adhoc Network (MANETs). The performance of VANET are affected by factors such as mobility, vehicle density and environmental condition. Provisioning smart infotainment application on such network is challenging and efficient MAC is required. Recently many Medium Access Control (MAC) based approaches adopting Time Division Medium Access (TDMA) and Carrier Sense Medium Access or Collision Avoidance (CSMA/CA) has been presented for VANET. The simulation outcome of exiting approaches shows that TDMA based approach outperforms CSMA/CA based approaches. However, TDMA based approaches incurs bandwidth wastages. To address, cognitive radio techniques is adopted by existing research. However, it incurs computation overhead and varied environmental condition such as urban, rural and highway are not considered. This work present a decentralized adaptive MAC (DAMAC) that maximize system throughput and minimize collision. Experiment are conducted to evaluate performance of DAMAC over exiting approaches. The outcome shows significant over existing approaches.
\end{abstract}

\section{Keywords:}

802.11P, Cognitive Radio, MAC, Multi-Channel, VANET

\section{INTRODUCTION}

VANET resembles a similar characteristic of Mobile Ad-Hoc Network (MANET) where vehicle are mobile in nature and are controlled by road topologies. The objective of VANET is to provision driver and user with safe and reliable communication environment. VANET is self-organizing network that aims to enhance the safety and efficiency of transportation and also provide infotainment on the go over vehicle to infrastructure (V2I), vehicle to vehicle (V2V) and combination of both (V2X). The architecture of VANET communication is shown in Fig.1. The U.S. Federal Communications Commission (FCC) allotted $75 \mathrm{MHz}$ frequency of licensed band in $5.9 \mathrm{GHz}$ for dedicated short range communication (DSRC), under IEEE 802.11p and IEEE 1609 standards for both safety and non-safety application under VANET environment. As a result various application has been presented in recent times. In the period of $5 \mathrm{G}$ era, VANET is been used in Internet of vehicles (IoVs) to provision ubiquitous smart infotainment services such as vehicular cyber-physical system [2], vehicular cloud networking [3].

To provision the above application requirement, the beaconing message should be transmitted over control channel $(\mathrm{CCH})$ with less latency reliably so that the VANET can assure the high speed and real-time data delivery application over service channel $(\mathrm{SCH})$. VANET are prone to high mobility hence periodic beaconing is required in order to obtain real time network related information in a cooperative manner. So it is quite a challenging task to design MAC. Another issue is channel congestion in MAC layer due to high network density. There are very high density of device that transmit their status message in its transmission range, which incurs packet collision overhead and increase packet delivery latency. A maximum latency for provisioning safety-related application is $100 \mathrm{~ms}$ as suggested in [4]. In [5] stated that the packet delivery ratio should not be less than $90 \%$ and the experimental outcome presented in [6] shows the impact of high density on packet delivery ratio and latency. Therefore, QoS cannot be guaranteed, if the MAC design is not scalable.

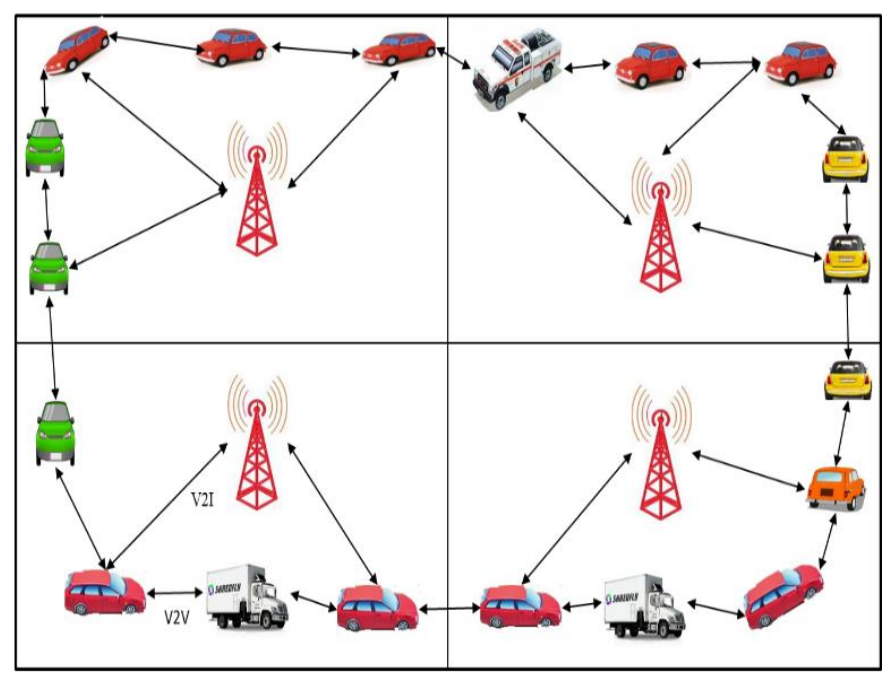

Fig.1. Architecture of VANET communication

To address the above research challenges, many MAC protocol is been presented. The CSMA/CA (Carrier Sense Multiple Access/Collision Avoidance) based 802.11p has been standardized as the standard MAC protocol for vehicular communication. However, if traffic density is high, it induces high collision probability, specifically for broadcasting packets [8]. CSMA/CA has the problem potentially unbounded channel access delay [9]. If the VANET device has multiple packets, it has to contend for multiple times. Further, 802.11p suffers from interference problem due to hidden terminals. Since it cannot use RTS (Request to Send) or CTS (Clear to Send) mechanism for broadcasting packets [10]. Considering this scenario, the collision of packet cannot even be identified right away. No exponential back-off methods can be used for broadcasting packet and the likelihood of packet collision is significantly very high [8].

To overcome the limitation of $802.11 p$, [11] presented a clustered based distributed multi-channel and mobility aware medium access control (DMMAC) design, which permits device to transmit their network related information to its cluster head every100ms. However, they are not efficient for safety related application [12]. Since, communication range is reduced to assure communication among cluster members. The extensive survey 
conducted by [13] shows that the clustered based MAC protocol performance are significantly affected by mobility of devices.

To improve performance and address the limitation of clustered network, various slotted MAC has been presented. In [14] and [15] presented a MAC based on RR-ALOHA where each node obtain status of slot reserved. Another widely adopted slotted MAC is TDMA-based approach [16], where time is divided into frames and each frames is composed of fixed number of time slots. Each vehicle access one slot in each frame. In [17] presented VeMAC using TDMA, in which each vehicle obtain one slot in a frame to transmit slot allocation information acknowledgement and data to its neighbor. However, their model incurs collision overhead due to hidden node problems. To address collision overhead in [18] presented prediction-based TDMA (PTMAC) which is distributed in nature. In [19] presented a self-sorting MAC which aided superior performance than [18] and scalable and cooperative MAC layer protocol (SCMAC) [20]. The model overcomes the bandwidth inefficiency of Space Division Multiple Access (SDMA) [21] and reduces collision for highway environment. However, their model did not considered maximizing system throughput which is a critical factor for provisioning infotainment application. To provision infotainment application [22] presented a MAC protocol adopting cognitive radio technique namely, enhanced non-cooperative cognitive division multiple access (ENCCMA). They combined FDMA (frequency division multiple access), TDMA and cognitive radio technique to design MAC for multi-channel network. The ENCCMA attained significant performance over state-of-art model. However, performance evaluation under different environment condition such as urban, rural and highway is not considered. To address the research challenges, this work present a Decentralized Adaptive MAC (DAMAC) protocol that minimize the collision and maximize the system throughput under different environment condition.

The contribution of research work is as follows: This work presented an optimal access mechanism to compute optimal objective function considering arbitrary vehicular traffic movement under single RSU. DAMAC is adaptive in nature considering varied environment condition such urban highway, and rural. The DMAC minimize collision and maximize system throughput under different environment condition [2]. The research outcome shows DAMAC is scalable irrespective of vehicle size.

The rest of the paper is organized as follows. Extensive research survey is carried out in section 2. In section 3 , the proposed decentralized distributed MAC model is presented. In penultimate section experimental study is carried out. The conclusion and future work is described in last section.

\section{PROPOSED DISTRIBUTED MAC DESIGN FOR V2I NETWORK}

This work presents decentralized medium access control design for V2I network which is distributed in nature. Let consider a MAC design, where time is segmented into identical slot time of length $\delta_{s}$ and there exist seamless synchronization among VANET subscribers or vehicles and the RSU (Road side unit). The total amount of time the subscriber stays in $x^{\text {th }} \mathrm{RSU}$ coverage area can be represented as:

$$
S_{x}=\left[\frac{2 K_{x}}{d \delta_{s}}\right]
$$

The $s^{\text {th }}$ time slot when subscriber is in range of $l^{\text {th }}$ RSU is obtained as follows,

$$
\mathcal{A}(x, \mathcal{S})=\sum_{h=0}^{x-1} S_{x}+\mathcal{S}_{x}, \forall \mathcal{S} \in\left\{1,2, \ldots, \mathcal{S}_{x}\right\}
$$

where, $S_{0}=0$. The set of time slots in $x^{\text {th }}$ RSU with respect to time line representation is $\mathcal{S}_{x}=\left\{\mathcal{A}(x, 1), \ldots, \mathcal{A}\left(x, T_{x}\right)\right\}$.

The optimal communication problem of a subscriber is considered as a finite-horizon sequential quality specifier (QS) problem. The QS time or iteration of the subscriber is:

$$
s=\mathbb{S}=\bigcup_{x \in X} \mathcal{S}_{x}=\bigcup_{x \in X}\left\{\mathcal{A}(x, 1), \ldots, \mathcal{A}\left(x, \mathcal{S}_{x}\right)\right\}
$$

where, $\mathbb{S}$ is the cumulated collection of all time slots in $X^{\text {th }}$ coverage range, where the mode $t \in T \in[0, T]$ depicts the outstanding size (in bits) of a packets to be sent. If we represent the number of subscribers in $x^{\text {th }}$ RSU coverage range as $v \in \mathcal{V}_{x}=$ $\{1, \ldots, V \uparrow, x\}$, then $l^{+ \text {ve }} \in \mathcal{L}_{x}=\left\{z_{x}(v): v \in \mathcal{V}_{x}\right\} \cdot \mathcal{L}_{x}=\left\{\mathcal{V}_{x}\right\}$.

The subscriber has two possible states at any modes $\left(t, l^{+\mathrm{ve}}\right)$, which can be represented as:

$$
m \in M=\{0,1\}
$$

where states $m=1$ infers that the subscriber agrees to request to transmit, and states $m=0$ the subscriber does not agrees to request to transmit.

The cost incurred at mode $\left(t, l^{\text {+ve }}\right)$ with instance $m$ at time slot $s \in \mathcal{S}_{x}$ in the $x^{\text {th }}$ coverage range is

$$
a_{s}\left(t, l^{+\mathrm{ve}}, m\right)=m z_{x}, \forall s \in \mathcal{S}_{x}
$$

Once the subscriber leaves the $x^{\text {th }}$ coverage area at instance $\mathcal{A}\left(X, S_{x}+1\right)$, the overhead incurred for subscriber for not completing his packet transmission is computed as follows

$$
\hat{a}_{\mathcal{A}\left(x, \mathcal{S}_{x}+1\right)}\left(t, l^{+v e}\right)=r(t)
$$

where, $r(t) \geq 0$ is a non-decreasing parameter of $t$ with $r(0) \geq 0$, which is associated with quality of service requirement of the application. Therefore, the cost of transmission incurred by the subscriber is composed of two things. Firstly, the communication cost in each time slot in Eq.(5) and the overhead incurred after leaving the $X^{\text {th }}$ coverage range in Eq.(6). The mode transitional likelihood $\left(\left(\bar{t}, l^{+v e}\right) \mid\left(t, l^{+v e}\right), m\right)$ is the likelihood that network will be in mode $\left(\bar{t}, l^{\overline{+v e}}\right)$ if states $m$ is taken at mode $\left(t, l^{+ \text {ve }}\right)$ at time slot $s \in \mathbb{T}$. The transition from $l^{+v e}$ to $l^{\overline{\overline{l v e}}}$ is not a determined by $t$ but determined by time $s$, thus we have

$$
l_{S}\left(\left(\bar{t}, l^{+v e}\right) \mid\left(t, l^{+v e}\right), m\right)=l_{S}\left(\bar{t} \mid\left(t, l^{+v e}\right), m\right) l_{s}\left(l^{\overline{+v e}} \mid l^{+v e}\right)
$$

With state $m=1$, we obtain, 


$$
l_{S}\left(\bar{t} \mid\left(t, l^{+v e}\right), 1\right)=\left\{\begin{array}{cl}
l^{+v e} & \text { if } \bar{t}=\left[t-b_{s} \delta s_{\text {packet }}\right], \\
1-l^{+v e} & \text { if } \bar{t}=t \\
0 & \text { otherwise }
\end{array}\right.
$$

where $[j]^{+}=\max \{0, j\}$. The case one and two depicts positive and negative data transmissions, respectively. With states $m=0$, thus we obtain:

$$
l_{S}\left(\bar{t} \mid\left(t, l^{+v e}\right), 0\right)=\left\{\begin{array}{l}
0 \text { if } \bar{t} \neq t \\
1 \text { otherwise }
\end{array}\right.
$$

where the remaining packets size to be transmitted will not change. The derivation of $l_{x}\left(l^{+\overline{+v e}} \mid l^{+v e}\right)$ are discussed in later subsection of this paper.

Let $\Delta_{s}: T \times L_{x} \rightarrow \mathcal{C}$ be the QoS specifier (QS) that defines the communication optimization of the subscriber at mode $\left(t, l^{+\mathrm{ve}}\right)$ at time slot $s \in S_{x}$ in the $x^{\text {th }}$ range of coverage. Now we express the objective function as QS covering all modes as $\left(\Delta_{s}\left(t, l^{+\mathrm{ve}}\right)\right.$, $\left.\forall t \in T, l^{+\mathrm{ve}} \in L_{x}, s \in S_{x} \forall x \in X\right)$. The mode at time slot $s$ if objective function $\mathcal{O}$ is used can be represented as $\left(t_{S}^{\mathcal{O}}, l_{S}^{+v e, \mathcal{O}}\right)$ and we consider (1) be the realistic set of $\mathcal{O}$. The subscriber aim at minimize the cost and satisfying objective function as an optimization problem.

$$
\min _{\mathcal{O} \in \mathcal{O}} Y_{\mathcal{O}}\left(T, l_{1}^{+v e}\right)\left[\sum_{x=1}^{\chi}\left[\begin{array}{c}
\sum_{k=1}^{S_{x} a_{\mathcal{A}(x, \mathcal{S})}}\left(\begin{array}{l}
t_{\mathcal{A}(x, \mathcal{S})}^{\mathcal{O}}, \\
l_{l(x, \mathcal{S})}^{+v e,}, \\
\Delta_{\mathcal{A}(x, \mathcal{S})}\left(t_{\mathcal{A}(x, \mathcal{S})}^{\mathcal{O}}\right) \\
a_{\mathcal{A}\left(\chi, \mathcal{S}_{\chi}+1\right)}\left(t_{\mathcal{A}\left(\chi, \mathcal{S}_{\chi}+1\right)}^{\mathcal{O}}, l_{l\left(\chi, \mathcal{S}_{\chi}+1\right)}^{+v e, \mathcal{O}}\right)
\end{array}\right]+
\end{array}\right]\right.
$$

where $Y\left(\mathcal{O}, l_{1}^{+v e}\right)$ represent the probability with respect to likelihood distribution by objective function $\mathcal{O}$ with an initial mode $\left(T, l_{1}^{+v e}\right)$ at time slot $s=\mathcal{A}(1,1)=1$.

Let consider that there exist only one $R S U$ and precise traffic pattern of subscriber is not known and Poisson process of subscriber arrival of $\alpha$ is considered. The transition likelihood of $l^{+\mathrm{ve}}$ is obtained as follows:

$$
\begin{gathered}
l_{S}\left(l^{+v e^{\prime}} \mid l^{+v e}\right)=l_{S}(l(\bar{v}) \mid l(v))=l_{S}(v \mid v) \\
l_{S}\left(l^{+v e^{\prime}} \mid l^{+v e}\right)=\left\{\begin{array}{cl}
\frac{(\alpha \delta s)^{\bar{v}-v+n_{s+1}}}{\left(\bar{v}-v+n_{s+1}\right) ! \beta_{s}(v)} & \text { if } v-n_{s+1} \leq \bar{v} \leq V_{\uparrow} \\
0 & \text { otherwise }
\end{array}\right.
\end{gathered}
$$

where, $\beta_{s}(v)=\sum_{i=0}^{V_{\uparrow}-v+n_{s+1}} \frac{(\alpha \delta s)^{i}}{i !}$ is a normalization function. Since $l^{\text {+ve }}$ is a rigorously reducing function of $v$, there is one-to-one mapping among $l^{+\mathrm{ve}}$ and $v$ as depicted in the first two equalities in Eq. (11) and the third equalities depicts the likelihood with $\bar{v}$ $v+b_{s+1}$ arrival due to Poisson process and $n_{s+1}$ deterministic displacement at instance $s+1 . \bar{v}$ is upper bounded by $V_{\uparrow}$ and lower bounded by $v-n_{s+1} \geq 0$ when there is no subscriber arrival.
The problem of Eq.(10) considering $R S U=\{1\}$ can be simplified as follows:

$$
\min _{\mathcal{O} \in \mathbb{O}} Y_{\mathcal{O},\left(T, l_{1}^{+v e}\right)}\left[\begin{array}{c}
\sum_{s=1}^{s} a_{s}\left(\left(t_{s}^{\mathcal{O}}, l_{s}^{+v e, \mathcal{O}}, \Delta_{s}\left(t_{s}^{\mathcal{O}}, l_{s}^{+v e, \mathcal{O}}\right)\right)\right)+ \\
\hat{c}_{T+1}\left(t_{S+1}^{\mathcal{O}}, l_{S+1}^{+v e, \mathcal{O}}\right)
\end{array}\right]
$$

Let $d_{s}\left(t, l^{+v e}\right)$ be the minimal projected total cost that the subscriber has to pay for contention from time $s$ to time $S+1$ when it is range of coverage considering network is in mode $\left(t, l^{+v e}\right)$ before the decision at time slot $s \in \mathcal{S}$. The optimization of minimal projected total cost at different modes for $s \in \mathcal{S}$ is as follows:

$$
d_{S}\left(t, l^{+v e}\right)=\min _{m \in M}\left\{\gamma_{S}\left(t, l^{+v e}, m\right)\right\}
$$

where

$$
\begin{gathered}
\gamma_{S}\left(t, l^{+v e}, m\right)=a_{S}\left(t, l^{+v e}, m\right)+ \\
\sum_{t \in T} \sum_{l^{+v e^{\prime}} \in \mathcal{L}} l_{S}\left(\left(\bar{t}, l^{+v e^{\prime}}\right) \mid\left(\bar{t}, l^{+v e}\right), c\right) d_{S+1}\left(\bar{t}, l^{+v e^{\prime}}\right) \\
\gamma_{S}\left(t, l^{+v e}, m\right)=c u+\sum_{l^{+v e^{\prime}} \in \mathcal{L}} l_{s}\left(l^{+v e^{\prime}} \mid l^{+v e}\right) \\
{\left[\begin{array}{l}
\left.c l^{+v e} d_{s+1}\left(\left[\bar{t}-b_{s} \delta_{s_{\text {packet }}}\right]^{*}, l^{+v e^{\prime}}\right)+\right] \\
\left(1-c l^{+v e}\right) d_{s+1}\left(t, l^{+v e^{\prime}}\right)
\end{array}\right]}
\end{gathered}
$$

The first term in Eq.(14) indicates the actual cost of selecting $c$ for the remaining time slots in coverage range and the second term in Eq.(14) indicates the future cost expected for selecting $c$ for the remaining time slots in coverage range. The Eq.(15) computes Eq.(14) directly by using Eq.(7), Eq.(8) and Eq.(9). For instance period $s=S+1$, we possess that limiting factor that,

$$
d_{s+1}\left(t, l^{+v e}\right)=\hat{a}_{s+1}\left(t, l^{+\mathrm{ve}}\right)=r(t)
$$

The value of $\gamma_{s}\left(t, l^{+v e}, m\right), \forall s \in S$, can be computed as follows,

$$
\begin{aligned}
& \gamma_{S}\left(t, l^{+v e}, m\right)=m u+ \\
& \sum_{g=0}^{v_{\uparrow}-n+n_{S}} \frac{(\alpha \delta s)^{g}}{g ! \beta_{S}(v)} \times\left[\begin{array}{l}
m l^{+v e} d_{s+1}\left(\begin{array}{c}
{\left[t-b_{S} \delta s_{\text {packet }}\right]^{*},} \\
z\left(v+g-n_{s+1}\right)
\end{array}\right)+ \\
\left(1-m l^{+v e}\right) d_{s+1}\left(t, z\left(v+g-n_{s+1}\right)\right)
\end{array}\right],
\end{aligned}
$$

where $v=\mathrm{z}^{-1}\left(l^{+\mathrm{ve}}\right)$ is the vehicle density in coverage range of RSU. The outcome follows directly by computing Eq.(15) using Eq.(11). Instinctively, the minimal projected cost $d_{S}\left(t, l^{\text {+ve }}\right)$ must be smaller when outstanding packet size t to be sent is smaller which can be assured when $d_{S}\left(t, l^{\text {+ve }}\right)$ is a non-decreasing parameter in $t, \forall l^{+\mathrm{ve}} \in \mathcal{L}, s \in \mathcal{S}$. Therefore, the optimal objective function $\mathcal{O}^{*}$ is obtained as follows,

$$
\mathcal{O}=\left(\Delta_{S}^{*}\left(t, l^{+\mathrm{ve}}\right)\right), \forall t \in T, l^{+\mathrm{ve}} \in \mathcal{L}, s \in \mathcal{S},
$$

where,

$$
\Delta_{S}^{*}\left(t, l^{+v e}\right)=\arg \min _{m \in M}\left\{\gamma_{S}\left(t, l^{+v e}, m\right)\right\} .
$$

The objective parameter $\mathcal{O}^{*}$ is an optimal solution of problem Eq.(12). In next section experimental study is carried out to evaluate the performance of proposed model over existing model. 


\section{SIMULATION RESULT AND ANALYSIS}

The experiment are conducted using windows 10 enterprises operating system, intel i7 class, 64bit, quad core processor, 12GB RAM, 4GB dedicated NVIDIA CUDA graphic card. The existing ENCCMA [1] and proposed DAMAC is implemented using C\# programming language, .net visual studio 4.0 framework using SIMITS simulator [1]. The experiment are conducted to evaluate the performance of DMAC over ENCCMA in term of collision and throughput achieved considering different environmental condition such as urban highway and rural. The radio propagation model for different environment are obtained from [23] which is incorporated in to SIMITS simulator. The simulation parameter used for experimental study are shown in Table.1.

Table.1. Simulation parameter considered

\begin{tabular}{|c|c|}
\hline Network Parameter & Value \\
\hline Network Size & $60 \mathrm{~m} \times 60 \mathrm{~m}$ \\
\hline Number of Vehicles & 20,40 and 60 \\
\hline Modulation scheme & QAM-64 \\
\hline Number of Frequency Channels & 7 \\
\hline Number of time slots & $8 \mu \mathrm{s}$ \\
\hline Bandwidth & $27 \mathrm{Mbps}$ \\
\hline Mobility of devices & 4 cycle per frame \\
\hline Coding rate & 0.75 \\
\hline Message information size & 27 bytes \\
\hline Environment used & Rural, Highway and Urban \\
\hline MAC used & ENCCMA and DAMAC \\
\hline
\end{tabular}

\subsection{COLLISION PERFORMANCE}

Experiments are conducted to evaluate the collision performance of proposed DAMAC over ENCCMA considering varied vehicles. The vehicles is varied as 20,40 and 60 and each vehicle is moving at speed of 4 cycle per frame. The Fig.2, Fig.3 and Fig.4 show the collision performance of DAMAC over ENCCMA for rural, highway and urban environment considering varied vehicles.

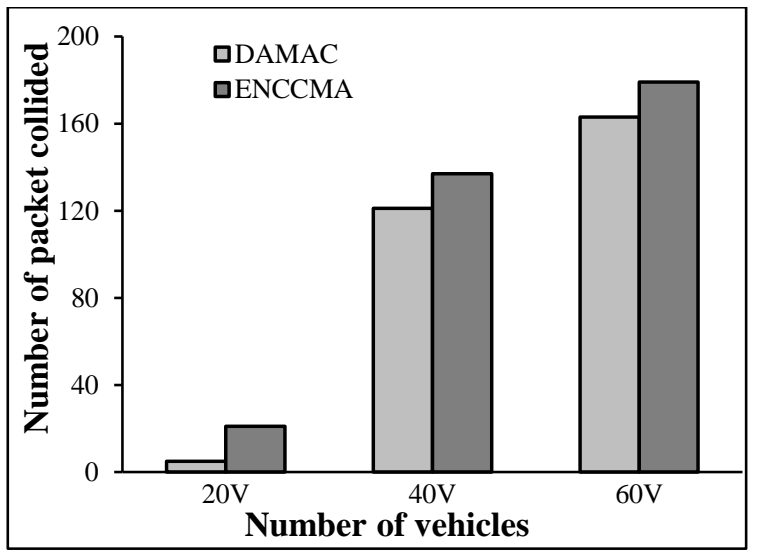

Fig.2. Collision performance for urban environment considering varied vehicle

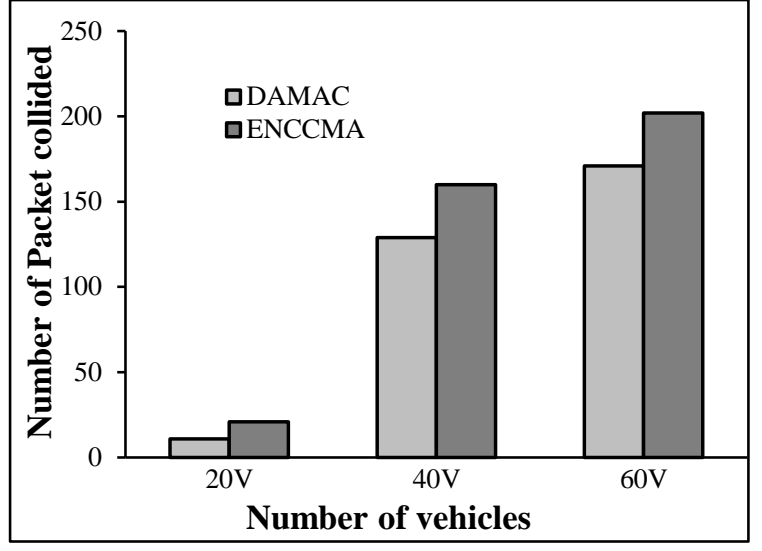

Fig.3. Collision performance for highway environment considering varied vehicle

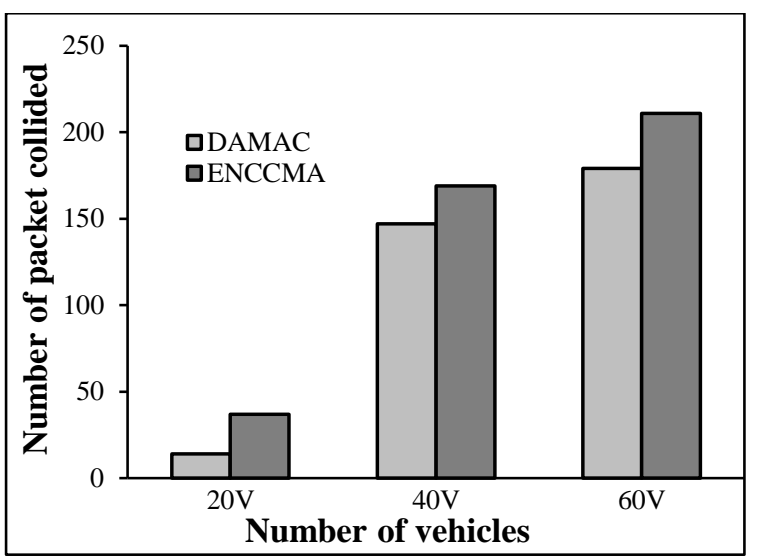

Fig.4. Collision performance for rural environment considering varied vehicle

The outcome shows that when vehicle size is increased the packet collision increases for both DAMAC and ENCCMA. From Fig. 2 it can be seen DAMAC reduces collision by $76.19 \%$, $11.67 \%$ and $8.93 \%$ for 20,40 and 60 vehicles respectively, over ENCCMA for urban environment. From Fig.3 it can be seen DAMAC reduces collision by $47.61 \%, 19.375 \%$ and $15.34 \%$ for 20, 40 and 60 vehicles respectively, over ENCCMA for highway environment. From Fig.4 it can be seen DAMAC reduces collision by $62.16 \%, 13.01 \%$ and $15.16 \%$ for 20,40 and 60 vehicles respectively, over ENCCMA for rural environment. An average reduction of $32.26 \%, 27.44 \%$ and $30.11 \%$ is achieved DAMAC over ENCCMA for urban, highway and rural environment respectively.

\subsection{THROUGHPUT PERFORMANCE}

Experiments are conducted to evaluate the throughput performance of proposed DAMAC over ENCCMA considering varied vehicles. The vehicles is varied as 20,40 and 60 and each vehicle is moving at speed of 4 cycle per frame. The Fig.5, Fig.6 and Fig.7 show the throughput performance of DAMAC over ENCCMA for urban, highway and rural environment considering varied vehicles. The outcome shows that when vehicle size is increased the throughput increases for both DAMAC and ENCCMA. From Fig.5 it can be seen DAMAC improves throughput by $16.79 \%, 8.39 \%$ and $7.6 \%$ for 20,40 and 60 vehicles 
respectively, over ENCCMA for urban environment. From Fig.6 it can be seen DAMAC improves throughput by $14.64 \%, 22.85 \%$ and $20.62 \%$ for 20,40 and 60 vehicles respectively, over ENCCMA for highway environment. From Fig.7 it can be seen DAMAC improves throughput by $18.98 \%, 16.06 \%$ and $14.33 \%$ for 20, 40 and 60 vehicles respectively, over ENCCMA for rural environment. An average improvement of $24.71 \%, 19.87 \%$ and $13.15 \%$ is achieved DAMAC over ENCCMA for urban, highway and rural environment respectively.

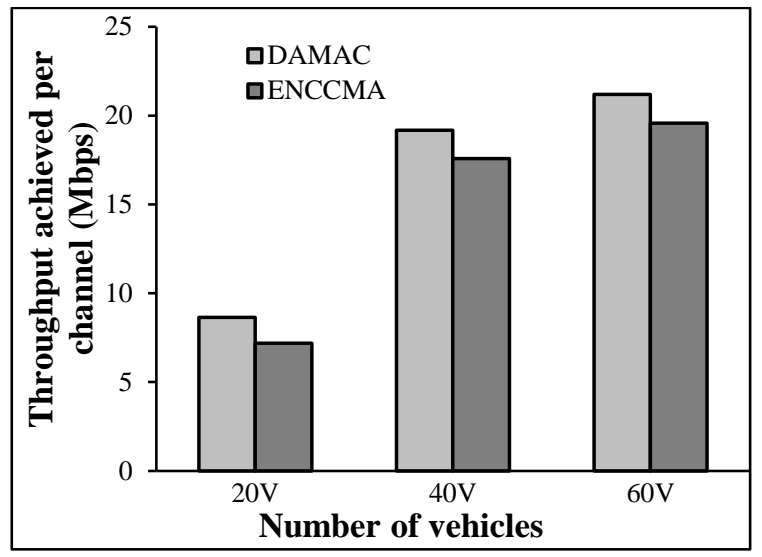

Fig.5. Throughput performance for urban environment considering varied vehicle

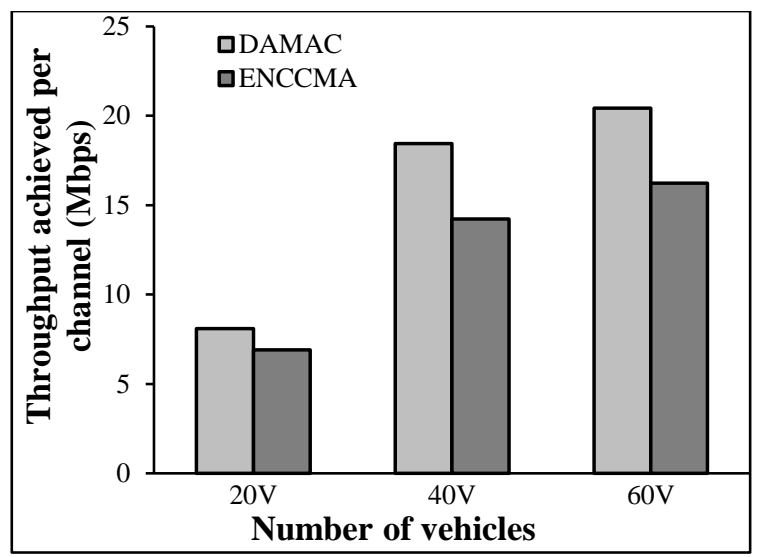

Fig.6. Throughput performance for highway environment considering varied vehicle

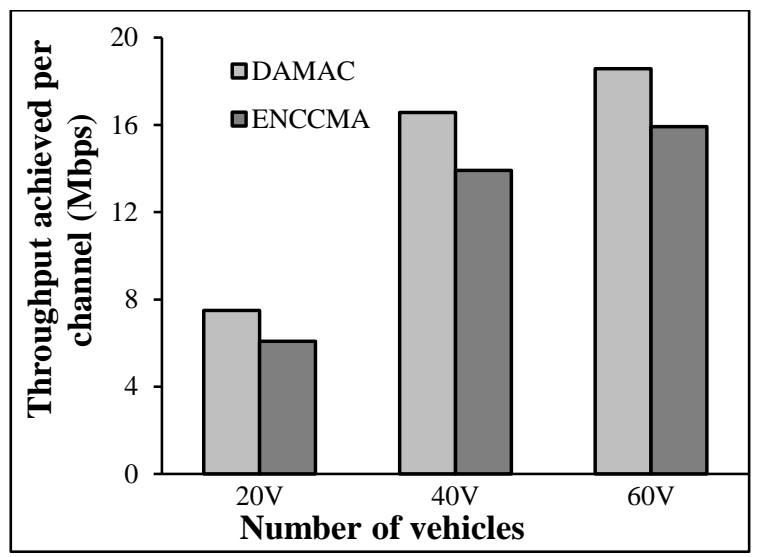

Fig.7. Throughput performance for rural environment considering varied vehicle

\section{CONCLUSIONS}

This work presented an efficient decentralized adaptive MAC design to maximize system throughput and minimize collision. The DAMAC aims to reduce the cost of data access (i.e., utilizing bandwidth more efficiently) for vehicle user. Our model achieves an optimal solution for the research objectives. Experiment are conducted to evaluate DAMAC performance over ENCCMA in term of collision and throughput achieved. The outcome shows, DAMAC reduces collision by $32.26 \%, 27.44 \%$ and $30.11 \%$ for urban, highway and rural environment respectively, over ENCCMA. DMAC improves throughput by $10.93 \%, 19.37 \%$ and $16.48 \%$ for urban, highway and rural environment respectively, over ENCCMA. The overall result achieved show that the DAMAC model is scalable irrespective of vehicle size and environmental condition. The future work would consider evaluation under varied network size and modulation scheme.

\section{REFERENCES}

[1] Mario Manzano, Felipe Espinosa; Ning Lu, Xuemin Shen, J.W. Mark and Fuqiang Liu, "Cognitive Self-Scheduled Mechanism for Access Control in Noisy Vehicular Ad Hoc Networks", Mathematical Problems in Engineering, Vol. 2015, pp. 1-11, 2015.

[2] D. Jia, K. Lu, J. Wang, X. Zhang and X. Shen, "A Survey on Platoon- Based Vehicular Cyber-Physical Systems", IEEE Communications Surveys and Tutorials, Vol. 18, No. 1, pp. 263-284, 2016.

[3] E. Lee, E.K. Lee, M. Gerla and S.Y. Oh, "Vehicular Cloud Networking: Architecture and Design Principles", IEEE Communications Magazine, Vol. 52, No. 2, pp. 148-155, 2014.

[4] S. Grafling, P. Mahonen and J. Riihijarvi, "Performance Evaluation of IEEE 1609 Wave and IEEE 802.11p for Vehicular Communications", Proceedings of $2^{\text {nd }}$ International Conference on Ubiquitous and Future Networks, pp. 344-348, 2010.

[5] Standard Specification for Telecommunications and Information Exchange Between Roadside and Vehicle Systems $5 \mathrm{GHz}$ Band Dedicated Short Range Communications (DSRC) Medium Access Control (MAC) and Physical Layer (PHY) Specifications, Available at: https://www.astm.org/Standards/E2213.htm

[6] K.A. Hafeez, L. Zhao, B. Ma and J.W. Mark, "Performance Analysis and Enhancement of the DSRC for VANETs Safety Applications", IEEE Transactions on Vehicular Technology, Vol. 62, No. 7, pp. 3069-3083, 2013.

[7] S. Neelambike and J. Chandrika, "An Efficient Environmental Model Considering Environmental Factor for V2I Application Services", Proceedings of IEEE International Conference on Computational Intelligence and Computing Research, pp. 1-6, 2015.

[8] T.V. Nguyen, F. Baccelli, Kai Zhu, S. Subramanian and Xinzhou Wu, "A Performance Analysis of CSMA based Broadcast Protocol in VANETs", Proceedings of IEEE International Conference on Communication Technology, pp. 2805-2813, 2013.

[9] M.I. Hassan, H.L. Vu and T. Sakurai, "Performance Analysis of the IEEE 802.11 Mac Protocol for DSRC Safety 
Applications", IEEE Transactions on Vehicular Technology, Vol. 60, No. 6, pp. 3882-3896, 2011.

[10] Quincy Tse, Weisheng $\mathrm{Si}$ and J. Taheri, "Estimating Contention of IEEE 802.11 Broadcasts based on Inter-Frame Idle Slots", Proceedings of IEEE $38^{\text {th }}$ Conference on Local Computer Networks Workshops, pp. 120-127, 2013.

[11] K.A. Hafeez, L. Zhao, J.W. Mark, X. Shen and Z. Niu, "Distributed Multichannel and Mobility-Aware Clusterbased MAC Protocol for Vehicular Adhoc Networks", IEEE Transactions on Vehicular Technology, Vol. 62, No. 8, pp. 3886-3902, 2013.

[12] Y. Yao, X. Zhou and K. Zhang, "Density-Aware Rate Adaptation for Vehicle Safety Communications in the Highway Environment", IEEE Communications Letters, Vol. 18, No. 7, pp. 1167-1170, 2014.

[13] C. Cooper, D. Franklin, M. Ros, F. Safaei and M. Abolhasan, "A Comparative Survey of VANET Clustering Techniques”, IEEE Communications Society, pp. 657-681, 2016.

[14] R. Scopigno and H.A. Cozzetti, "Mobile Slotted Aloha for VANETs", Proceedings of IEEE $70^{\text {th }}$ Vehicular Technology Conference Fall, pp. 1-5, 2009.

[15] F. Han, D. Miyamoto and Y. Wakahara, "RTOB: A TDMAbased MAC Protocol to Achieve High Reliability of OneHop Broadcast in VANET", Proceedings of IEEE
International Conference on Pervasive Computing and Communications Workshops, pp. 87-92, 2015.

[16] M. Hadded, P. Muhlethaler, A. Laouiti, R. Zagrouba and L.A. Saidane, "TDMA-based MAC Protocols for Vehicular Ad Hoc Networks: A Survey, Qualitative Analysis, and Open Research Issues", IEEE Communications Surveys and Tutorials, Vol. 17, No. 4, pp. 2461-2492, 2015.

[17] H.A. Omar, W. Zhuang and L. Li, "VeMAC: A TDMAbased MAC Protocol for Reliable Broadcast in VANETs", IEEE Transactions on Mobile Computing, Vol. 12, No. 9, pp. 1724-1736, 2013.

[18] X. Jiang and D.H. Du, "PTMAC: A Prediction-based TDMA MAC Protocol for Reducing Packet Collisions in VANET", IEEE Transactions on Vehicular Technology, Vol. 65, No. 11, pp. 9209-9223, 2016.

[19] Z. Shen, X. Zhang, M. Zhang, W. Li and D. Yang, "SelfSorting-Based MAC Protocol for High-Density Vehicular Ad Hoc Networks", IEEE Access, Vol. 5, pp. 7350-7361, 2017.

[20] Y. Cao, H. Zhang, X. Zhou and D. Yuan, "A Scalable and Cooperative MAC Protocol for Control Channel Access in VANETs", IEEE Access, Vol. 5, pp. 9682-9690, 2017.

[21] Z. Doukha and S. Moussaoui, "A SDMA-based Mechanism for Accurate and Efficient Neighborhood Discovery Link Layer Service", IEEE Transactions on Vehicular Technology, Vol. 65, No. 2, pp.1-11, 2015. 